\title{
¿SON IGUALES TODOS LOS SISTEMAS EMPLEADOS PARA CORREGIR LA INCONTINENCIA URINARIA MEDIANTE MALLAS LIBRES DE TENSIÓN?
}

\author{
I. GALMÉS BELMONTE*, E. DÍAZ GÓMEZ**
}

*Unidad de Uroginecología y Patología del Suelo Pelviano. Hospital Universitario Santa Cristina. Madrid. **Departamento de Urología. Unidad de Suelo Pelviano. Hospital Universitario Gregorio Marañón. Madrid.

Actas Urol Esp. 28 (7): 487-496, 2004

\section{RESUMEN}

¿SON IGUALES TODOS LOS SISTEMAS EMPLEADOS PARA CORREGIR LA INCONTINENCIA URINARIA MEDIANTE MALLAS LIBRES DE TENSIÓN?

Desde el año 1996 en que Ulmsten describe la técnica del TVT (Tension-free vaginal tape) para la corrección de la Incontinencia Urinaria de Esfuerzo (IUE), han sido numerosas las variantes descritas y los dispositivos existentes en el mercado que tratan de reproducir la técnica original. Sin embargo, los resultados no van a depender exclusivamente de la técnica quirúrgica sino también de otros dos factores fundamentales que son:

1. Características y tipo de malla utilizada.

2. Sistema y vía de implantación de la misma.

Las características de la malla empleada posiblemente sean tan importantes o más que la propia técnica quirúrgica. No es posible extrapolar los datos relativos al TVT para otros dispositivos. Sería necesario realizar estudios randomizados en los que se comparen las diferentes técnicas para poder hacer afirmaciones semejantes. La vía de implantación tiene también una gran importancia en los resultados obtenidos.

En el presente trabajo se hace una revisión de las características de las mallas y sus propiedades así como de las diferentes vías de abordaje y colocación de las mismas. Se analiza también la influencia de cada uno de estos factores sobre los resultados quirúrgicos. Finalmente se repasan los principales dispositivos existentes en el mercado, las propiedades de la malla y del sistema de implantación de cada uno de ellos.

PALABRAS CLAVE: TVT. Incontinencia urinaria de esfuerzo femenina. Cirugía de la incontinencia. Características de las mallas.

\section{ABSTRACT}

THE DEVICES USED TO CORRECT THE URINARY INCONTINENCE BY TENSION-FREE MESHES. ARE ALL THEM EQUALS?

Since 1996, when Ulmsten described the TVT procedure (Tension-free Vaginal Tape) for correction of women urinary stress incontinence, a large number of different devices have been manufactured with that purpose. Results depend not only of the surgical procedure but also of two principal factors:

1. Characteristics and properties of the mesh.

2. System and way for the implantation.

Properties of the mesh used are probably as important as surgical technique. It's not possible to assume that results achieved with the TVT device will be similar with other meshes. Further randomized studies will be necessary to make these affirmations.

In this paper we analyse properties of the mesh, characteristics of the implantation system and the influence of those factors in the surgical results. Finally, we describe some of the devices available and the mesh characteristics of each one.

KEY WORDS: TVT. Woman urinary stress incontinence. Incontinence surgery. Mesh characteristics. 


\section{GE NERALIDADES SOBRE LAS MALLAS}

1.1. Características físicas de las mallas

1.1.1. Material de fabricación: dependiendo del material de fabricación, las mallas se pueden clasificar de la siguiente forma:

- Mallas biológicas: que según su origen pueden ser:

- Autólogas: material biológico obtenido del propio paciente (aponeurosis de los rectos; tendón de fascia lata; mucosa vaginal). La utilización de este tipo de injertos supone un incremento de la morbilidad y los resultados resultan frecuentemente poco satisfactorios $^{1}$. Actualmente su uso es muy limitado y cada vez más restringido.

- Aloinjertos: material biológico obtenido de otros humanos (dura madre, fascia lata). Este tipo de material presenta un pequeño riesgo de transmisión de enfermedades causadas por priones, infecciones por HIV, virus lentos, etc. ${ }^{1}$. Por otra parte, los aloinjertos pueden mantener una antigenicidad residual suficiente para desencadenar una respuesta inmune que lo destruya ${ }^{1}$. Recientemente se han empezado a utilizar aloinjertos acelulares con los que resulta mucho más infrecuente el riesgo de desarrollar una respuesta inmune.

- Xenoinjertos: material biológico obtenido de animales (intestino delgado porcino, dermis porcina, pericardio bovino). Con este tipo de injertos el rechazo inmunológico puede ser incluso mayor que con los aloinjertos. También es posible la transmisión de enfermedades causadas por priones.

- Mallas sintéticas: polipropileno (prolene, marlex, atrium), ácido poliglicólico (dexon), poliglactin 910 (vicryl), PTFE (gore-tex, Teflon), polietileno (mersilene), etc.

1.1.2. Estructura del material: hace referencia al carácter monofilamento o multifilamento de cada una de las hebras de la malla (Figs. 1 y 2).

- Es de tipo monofilamento: polipropileno (habitualmente).

- Son de tipo multifilamento: PTFE, polietileno, ácido poliglicólico, poliglactin 910.

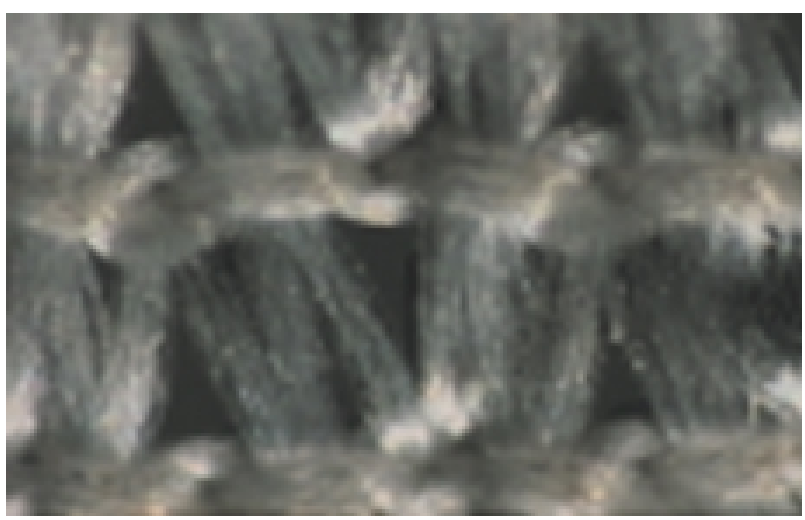

FIGURA 1. Malla multifilamento.

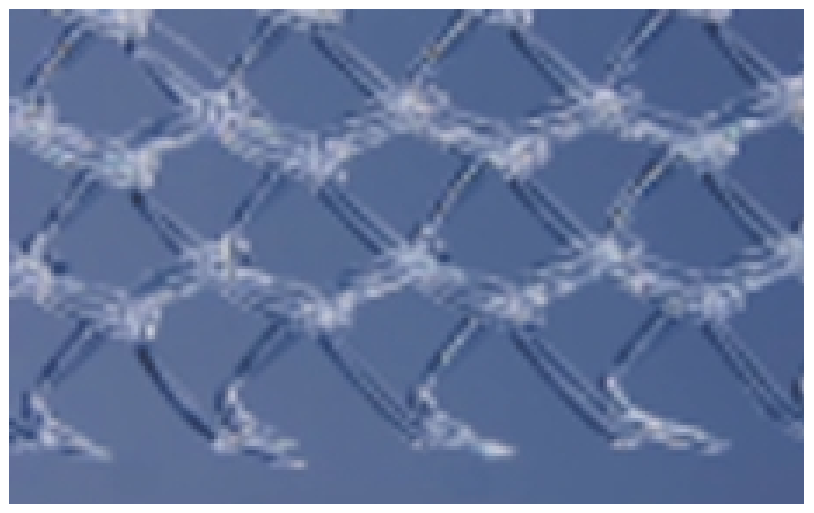

FIGURA 2. Malla monofilamento.

1.1.3. Tamaño de los poros: el tamaño de los poros de cada uno de los filamentos que componen la malla resulta uno de los factores más importantes a tener en cuenta. Estudios experimentales han demostrado que el tamaño de los poros influye en aspectos tan importantes como $^{1}$ :

- Resistencia a las infecciones.

- Flexibilidad de la prótesis.

- Infiltración por los fibroblastos: cicatrización, integración en tejido circundante, formación de seromas.

- Anclaje mecánico de la malla.

- Angiogénesis.

Atendiendo a esta propiedad las mallas se clasifican en 3 grupos ${ }^{2}$ :

- Macroporosas: los poros de los filamentos tendrán más de $75 \mu \mathrm{m}$ de diámetro menor.

- Microporosas: cuando el poro es <10 $\mu \mathrm{m}$.

- Poros submicroscópicos: cuando el tamaño del poro es $<1 \mu \mathrm{m}$.

Teniendo en cuenta la estructura y la porosidad del material, las mallas se clasifican en cuatro grandes grupos (Clasificación de Amid) ${ }^{2}$ : 
- Tipo I (totalmente macroporosas): aquellas mallas monofilamento y macroporo.

- TVT

- SPARC

- Gynemesh

- Tipo II (totalmente microporosas):

- Mallas de Goretex

- Tipo III (mallas macroporosas con elementos microporosos): aquellas mallas macroporosas que tienen elementos micoporosos en su estructura (por ejemplo multifilamentos).

- IVS

- Mycromesh

- Tipo IV (mallas con poro submicroscópico).

1.1.4. Estructura del entramado: atendiendo a la estructura del entramado, podemos diferenciar tres grandes grupos:

- Mallas tricotadas: los diferentes filamentos van entrelazados y anudados entre sí.

- Mallas tejidas: los filamentos van entrelazados en dos direcciones, como fabricadas en un telar. Generalmente se trata de mallas muy tupidas con una gran densidad de material.

- Mallas termocoaguladas: cuando el entramado de los filamentos se consigue al fundirlos con calor.

1.1.5. Densidad del material (porosidad): la densidad hace referencia a la cantidad de material por unidad de superficie. La densidad va a depender tanto del diámetro de los hilos que forman la malla como de la proximidad entre los mismos $^{3}$. La porosidad se cuantifica en porcentaje (proporción entre superficie del agujero/superficie total). Esta propiedad va a influir en la respuesta inflamatoria así como en la probabilidad de formar seromas o de causar erosiones. Se ha demostrado que la intensidad del infiltrado de macrófagos y el grosor de la cápsula fibrosa formada dependen directamente de la densidad de la malla ${ }^{3,4}$. Según sea la porosidad, las mallas pueden clasificarse $\mathrm{en}^{4}$ :

- De alta densidad: mallas muy tupidas en las que apenas hay huecos entre los diferentes filamentos entrecruzados.

- De baja densidad: mallas con grandes huecos entre los filamentos.

- De densidad intermedia.

\subsection{Propiedades mecánicas de las mallas}

1.2.1. Resistencia: la resistencia de la malla se refiere a la capacidad para soportar tensiones sin romperse (Fig. 3). Por lo general se trata de una propiedad sobredimensionada en la mayor parte de los materiales existentes en el mercado, siendo, por tanto, un factor poco influyente.

Además de la resistencia propia de la malla, hay que tener en cuenta su resistencia a largo plazo tras la implantación en el paciente ${ }^{5}$. Esta propiedad dependerá de dos factores esenciales:

- Material de fabricación: absorbible o no absorbible ${ }^{6}$. En este sentido se ha podido comprobar que la utilización de mallas reabsorbibles no permite garantizar la solidez de la reparación a largo plazo, incrementándose los porcentajes de recidiva ${ }^{6}$.

- Integración de la malla con el tejido circundante: cuanto mayor sea la integración del entramado con el tejido fibroso, mayor será la resistencia del conjunto. Por el contrario, en caso de escasa integración o encapsulamiento, la resistencia será más reducida ${ }^{5}$. La resistencia final va a depender fundamentalmente del grado de depósito de fibras de colágeno maduro tipo $\mathrm{I}^{7}$.

1.2.2. Elasticidad: entendemos por elasticidad a la capacidad de elongación sin ruptura y recuperación posterior de la longitud inicial. Lo aconsejable es que una malla posea una buena elasticidad sobre todo inicial, para fuerzas de tracción pequeñas (que son las que va soportar fisiológicamente).

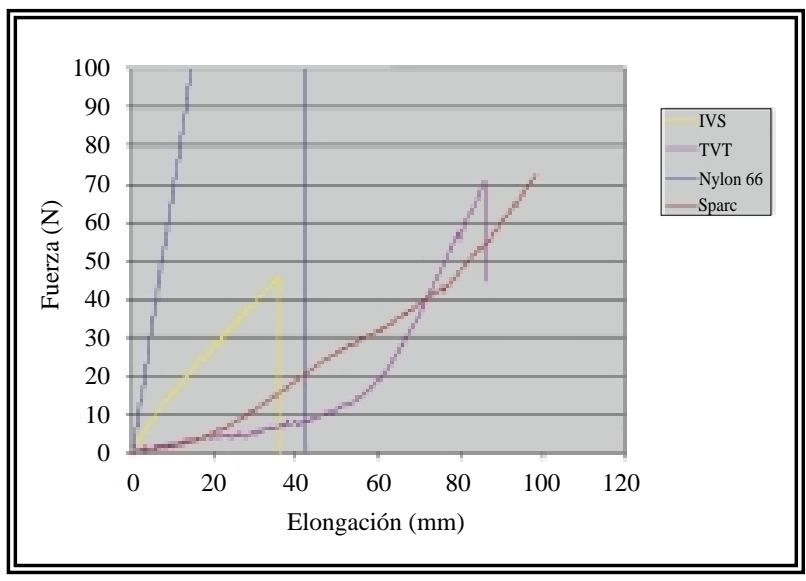

FIGURA 3 
Se trata de una propiedad cuya importancia no ha sido ampliamente aceptada hasta hace poco tiempo. En los últimos años se ha podido comprobar su relevancia, sobre todo en la cirugía del suelo pélvico. La falta de elasticidad de la cicatriz condiciona una mayor rigidez vaginal y puede causar molestias durante el coito, limitar la distensibilidad de la vejiga, provocar sintomatología miccional irritativa, etc. ${ }^{4}$.

La elasticidad va a depender no sólo de las características de la malla (porosidad, densidad, material de fabricación, estructura del entramado) sino también de su interacción con el huésped (tejido fibroso cicatricial resultante, integración con el mismo, etc. ${ }^{8}$. Se ha podido comprobar que la disposición de los haces de fibras colágenas adoptan una distribución paralela al entramado de filamentos de la malla cuando la densidad de ésta es baja. Esta disposición más regular del colágeno proporciona una mayor elasticidad a la cicatriz ${ }^{9}$.

La elasticidad de los tejidos naturales para una fuerza tensil de $16 \mathrm{~N}$ varía entre el $10 \mathrm{y}$ el $35 \%^{8}$. Dicha elasticidad es la que se debe intentar conseguir con los materiales implantados. Las mallas de polipropileno son las que mejor se adaptan a este comportamiento ${ }^{10}$.

1.2.3. Maleabilidad, ductilidad: es la capacidad de la malla para adaptarse a la superficie sobre la que se coloca, ajustándose a los relieves e irregularidades de dicha superficie.

\subsection{Comportamiento de las mallas una vez implantadas}

1.3.1. Capacidad de absorción del material: dependiendo del comportamiento del material, en relación a la capacidad para ser o no reabsorbido, las mallas pueden clasificarse en:

- Absorbibles: elaboradas con material que resulta digerido por el propio organismo receptor. Derivadas del ácido poliglicólico (vicryl, dexon).

- No absorbibles: Polipropileno (Prolene); ePTFE; Poliéster; composites.

- Parcialmente absorbibles: elaboradas con mezcla de materiales (absorbible y no absorbible), p. ej. Polipropileno y ac. Poliglicólico (Vypro II).
1.3.2. Resistencia frente a las infecciones: se trata de un factor fundamental a tener en cuenta y que va a depender de las siguientes características:

- Tamaño del poro del filamento: el macrófago tiene un tamaño aproximado de 16-20 $\mu \mathrm{m}$, los leucocitos 9-15 $\mu \mathrm{m}$ y las bacterias tienen un tamaño de alrededor de $1 \mu \mathrm{m}$. Los macrófagos y los leucocitos son incapaces de atacar a las bacterias cuando éstas se refugian en poros de menos de $10 \mu \mathrm{m}$ de diámetro. La influencia de este factor es muy grande, como ha podido ser demostrada en múltiples estudios ${ }^{11}$. La incidencia de infección publicada para mallas del tipo II / III de Amid varía entre el 9,6-50\% mientras que de es del 0\% para las tipo I. La infección del implante en mallas tipo I puede ser manejada mediante tratamiento antibiótico y drenaje de la herida. Por el contrario, en las mallas tipo II ó III es necesaria la retirada de la malla ${ }^{12}$.

- Además del tamaño de los poros, el espacio entre los filamentos en las mallas multifilamento puede llegar a ser de menos de 10 $\mu \mathrm{m}$. Es por ello que este tipo de mallas no se incluyen en le subtipo I de Amid (totalmente macroporosas) ${ }^{13}$.

- Rapidez del crecimiento del tejido fibroso y vascular. La rápida vascularización de la cicatriz y el incremento en la adhesión a los tejidos circundantes, reduciendo los espacios muertos, son factores que contribuyen decisivamente a disminuir el riesgo de infección ${ }^{12}$. La capacidad de angiogénesis y la formación de colágeno depende de la interacción de moléculas específicas de la matriz extracelular y factores de crecimiento con capacidad angiogénica con el material que forma la malla. Esta interacción resulta especialmente favorable en caso de mallas macroporosas tipo $\mathrm{I}^{14}$.

1.3.3. Bien tolerada, biocompatible, inerte: se habla de material biocompatible a aquel que causa una reacción favorable cuando se implanta en un ser vivo. No debe inducir una respuesta inflamatoria ni alérgica, ni carcinogénica. La tolerancia y biocompatibilidad de las mallas depende 
de dos factores fundamentales: el material de fabricación ${ }^{11,15}$ y la densidad del mismo. Cuanto mayor sea la densidad (malla más tupida) menor será su biocompatibilidad. Se ha podido comprobar que cuanto menor resulta la densidad de una malla, menor es la respuesta inflamatoria induci$\mathrm{da}^{9}$ (menor número de fenómenos de apoptosis y de proliferación celular; incremento en la expresión de factor citoprotector HSP70 ${ }^{16}$. Para que una malla sea biocompatible es necesario que:

- No induzca una reacción inflamatoria: una respuesta inflamatoria exagerada incrementa el riesgo de formación de seromas. Otro de los factores que facilitan la acumulación de un seroma es la existencia de espacios muertos entre la malla y el tejido circundante. Estos espacios surgen cuando la malla se integra lenta o escasamente con el tejido circundante y esta circunstancia depende, como ya hemos visto, del tamaño del poro y de la densidad del material ${ }^{12,15}$.

- No induzca una reacción alérgica: la reacción alérgica provocará el rechazo y la extrusión de la prótesis ${ }^{11}$.

- No induzca un crecimiento tumoral.

1.3.4. Adhesión al tejido circundante: la adhesión de la malla al tejido circundante va a depender de dos factores fundamentales:

- Inicialmente va a depender del "efecto velcro" de la malla, es decir, de la estructura del trenzado y de los filamentos que componen la propia malla. Esta propiedad es importante para mantenerla en su correcta posición mientras se produce su integración con el tejido circundante.

- Posteriormente la adhesión dependerá de la integración del tejido fibroso en la propia malla.

1.3.5. Retracción mínima: la retracción de las mallas es debida al tejido fibroso-cicatricial que inducen. En todos los casos se va a producir una cierta retracción que se estima será de entre el $10-20 \%{ }^{11}$. Por este motivo es obligado dejar siempre la malla sin ninguna tensión y suficientemente holgada. Cuanto menor sea la integración del entramado con el tejido fibroso, mayor será la retracción que experimente la cicatriz.

\section{GENERALIDADES SOBRE LA VÍA DE INSERCIÓN}

\subsection{Vía retropúbica}

Es la vía inicialmente descrita para la inserción del TVT en 1997. Previamente ya había sido ampliamente utilizada para la corrección quirúrgica de la IUE, habiéndose descrito múltiples procedimientos en los que se empleaban agujas para pasar los hilos de tracción desde la cavidad vaginal hasta la pared abdominal. Mediante este abordaje quirúrgico se pasan las agujas atravesando los ligamentos parauretrales, la fascia endopélvica, el espacio de Retzius y la pared abdominal. Se debe intentar que las agujas de inserción atraviesen estas estructuras lo más próximo posible a los huesos de la pelvis. Según sea la forma de pasar las agujas, existen descritas dos formas de abordaje: la inferior (desde vagina a pared abdominal) y la superior (desde pared abdominal a vagina). Los principales inconvenientes de la vía retropúbica son los siguientes:

- Perforación de la vejiga: circunstancia que resulta relativamente frecuente y que hace imprescindible la realización de un estudio endoscópico intraoperatorio. Se estima que el porcentaje de pacientes en las que se produce dicha lesión vesical es de alrededor del $6 \%{ }^{17}$. La perforación resulta especialmente frecuente en mujeres previamente operadas o muy delgadas. La perforación advertida y corregida apenas modifica el pronóstico ni la morbilidad del procedimiento.

- Lesión vascular: la lesión vascular es también relativamente frecuente, sobre todo la lesión de los vasos venosos prevesicales del espacio de Retzius. Mediante estudio ecográfico o determinación posquirúrgica de hemograma (hematocrito y hemoglobina) es posible encontrar un elevado porcentaje de pacientes en las que ha existido una hemorragia más o menos grande en dicho espacio. Algunas autores llegan a cifrar este porcentaje en hasta el $15 \%$ de los $\operatorname{casos}^{18}$, si bien solo en el $0,8 \%$ de los casos va a tener trascendencia clínica ${ }^{19,20}$. Las posibilidades de sangrado se incrementan cuando existen anastomosis venosas retropúbicas. Esta circunstancia es posible encontrarla en hasta 
$1 / 3$ de las mujeres. Para reducir las posibilidades de sangrado de este plexo venoso es aconsejable hacer los orificios de salida de las agujas a unos 3-4 cm de la línea media y dejar la vejiga rellena con unos 300 cc de suero durante 60-90 minutos tras la intervención. La vejiga llena ejercerá un efecto compresivo sobre dichas venas e impedirá el sangrado excesivo. Mucho más infrecuente es la lesión de vasos de mayor calibre, generalmente vasos iliacos. Hay descritos unos 20 casos en la literatura de lesión de vasos ilíacos ${ }^{21}$.

- Lesión de asas intestinales: se trata de una complicación muy infrecuente pero de una gran trascendencia clínica. Generalmente ocurre cuando las agujas se sacan en una posición alta en abdomen ${ }^{22,23}$.

Existen dos vías de abordaje fundamentales para la implantación de las mallas en una posición retropúbica:

2.1.1. Inferior (desde vagina a abdomen): cuando las agujas se insertan desde la cavidad vaginal hacia la pared abdominal. Recientemente se ha descrito un abordaje inferior prepúbico ${ }^{24}$, con el que se pretende evitar el riesgo de perforación vesical y de sangrado en Retzius al pasar las agujas por delante del hueso púbico, a través del tejido celular subcutáneo. De esta forma parece que el grado de sujeción de la malla es peor y las molestias postoperatorias son mayores. Se desconocen todavía los resultados a largo plazo con esta variación técnica.

2.1.2. Superior (desde abdomen a vagina): cuando las agujas se colocan desde la pared abdominal hacia la vagina. Esta forma de inserción tiene la ventaja de que resulta más difícil la lesión accidental de grandes vasos o de asas intestinales, pero plantea algunos problemas:

- Es necesaria una mayor incisión vaginal y una mayor disección parauretral.

- En pacientes obesas se dificulta la correcta posición de las agujas.

- La malla tiende a ser colocada en una posición más próxima al cuello vesical, lo que supone un incremento en la aparición de disuria posquirúrgica ${ }^{25}$.

\subsection{Vía transobturatriz}

La vía transobturatriz surge para tratar de evitar la perforación accidental de la vejiga y por tanto, la necesidad de realizar una exploración endoscópica intraoperatoria ${ }^{26,27}$. Las agujas se van a pasar desde la cara interna del muslo hasta la región vaginal parauretral a través del agujero obturador y los músculos puborrectales ${ }^{26}$. Se han descrito dos abordajes diferentes: desde el muslo hasta la vagina (técnica "out-in") y en dirección contraria ("in-out”). Este tipo de corrección reproduce la suspensión anatómica natural de la uretra. También parece que la incidencia de disuria en el postoperatorio es menor que con la vía retropúbica ${ }^{26}$. Sin embargo, tal y como se ha demostrado en algunos trabajos, la probabilidad de lesionar la vejiga no es nula, por lo que no resulta aconsejable eliminar la necesidad de una cistoscopia, sobre todo en los abordajes "outin"28. La utilización de un acceso "in-out", es decir, desde la uretra hacia el exterior, prácticamente anula la posibilidad de lesión vesical (haciendo innecesario el estudio endoscópico intraoperatorio) y permite una menor disección parauretral.

Los resultados, relativos a la corrección de la IUE a los 12 meses de evolución, parecen ser semejantes a los conseguidos con la vía retropúbica ${ }^{29}$. Sin embargo, para poder valorar la eficacia y los resultados a largo plazo de esta nueva vía de abordaje serán necesarios estudios futuros bien diseñados que comparen ambas técnicas en el tiempo.

\section{UTILIZACIÓN DE LOS SISTEMAS LIBRES DE TENSIÓN PARA CORREGIR LA IUE}

Son múltiples las opciones actualmente existentes en el mercado para la corrección quirúrgica de la IUE mediante sistemas de malla libre de tensión (Tabla 1). Sin embargo no todos los productos existentes son iguales y la elección de uno $\mathrm{u}$ otro debería hacerse sobre la base de criterios fundados. A continuación se hace una breve descripción de cada uno de ellos, comentando sus principales características y propiedades.

\subsection{TVT (Gynecare) (Fig. 4)}

Es la malla más ampliamente utilizada y por ello debe ser considerada el patrón de referencia 
TABLA 1

\begin{tabular}{|c|c|c|c|c|c|}
\hline & TVT & SPARC / MONARC & IVS & URATAPE / OBTAPE & URETEX \\
\hline Fabricante & Gynecare & AMS & Tyco & Porges - Mentor & Bard - Sofradin \\
\hline Composición & Polipropileno & $\begin{array}{c}\text { Polipropileno }+ \\
\text { Sutura absorbible (PGA) }\end{array}$ & Polipropileno & Polipropileno & Polipropileno \\
\hline Estructura filamento & Monofilamento & Monofilamento & Multifilamento & Monofilamento & Monofilamento \\
\hline Estructura malla & Tricotada & Tricotada & Tricotada & Termocoagulada & Tricotada \\
\hline Geometría malla & Cuadrilátero & Cuadrilátero & Triángulo & Cuadrado & $\begin{array}{c}\text { Cuadrilátero } \\
\text { (Diagonales: 1' x } \\
\text { 2'4 mm) }\end{array}$ \\
\hline $\begin{array}{l}\text { Porosidad (superficie } \\
\text { agujero / superficie } \\
\text { total) }\end{array}$ & $60 \%$ & $46 \%$ & $10 \%$ & $13 \%$ & $64 \%$ \\
\hline Tamaño poro & $>75 \mu \mathrm{m}$ & $>75 \mu \mathrm{m}$ & $<10 \mu \mathrm{m}$ & $<10 \mu \mathrm{m}$ & $>75 \mu \mathrm{m}$ \\
\hline Tipo Amid & Tipo I & Tipo I & Tipo III & Tipo II & Tipo I \\
\hline Peso $(\mathrm{g} / \mathrm{m} 2)$ & 95 & 120 & 84,2 & 98 & 87 \\
\hline Grosor (mm) & 0,65 & 0,69 & 0,38 & 0,55 & 0,63 \\
\hline Limite rotura & $84 \mathrm{~N}$ & $50 \mathrm{~N}$ & & $50 \mathrm{~N}$ & $76 \mathrm{~N}$ \\
\hline $\begin{array}{l}\text { Elasticidad } \\
(\% \text { elongación con } \\
15 \mathrm{~N})\end{array}$ & $92 \%$ & $44 \%$ & $12 \%$ & $8 \%$ & $54 \%$ \\
\hline Dimensiones & $1,1 \times 45 \mathrm{~cm}$ & $1,1 \times 35 \mathrm{~cm}$ & $0,6 \times 40 \mathrm{~cm}$ & $2 \times 60 \mathrm{~cm}$ & $1,1 \times 45 \mathrm{~cm}$ \\
\hline Vía implantación & $\begin{array}{l}\text { Abdominal; retropúbica; } \\
\text { transobturadora }\end{array}$ & $\begin{array}{l}\text { Abdominal; } \\
\text { transobturador }\end{array}$ & Retropúbica & Transobturadora & $\begin{array}{l}\text { Abdominal; } \\
\text { Retropúbica }\end{array}$ \\
\hline
\end{tabular}

para comparar al resto. Sus características fundamentales son las siguientes:

- Fabricada con polipropileno monofilamento (Prolene), tipo I de Amid. Basándose en los datos experimentales publicados, el polipropileno parece el material más adecuado para utilizar en las mallas libres de tensión empleadas para corregir la IUE ${ }^{2,12}$.

- Tricotada.

- Baja densidad, tiene una porosidad del $60 \%$.

- Elasticidad óptima: la malla posee una capacidad de elongación muy buena, sobre todo para tensiones de hasta $10 \mathrm{~N}$. Con dicha fuerza se consigue una elongación de 40 $\mathrm{mm}$. El límite de ruptura se alcanza para una fuerza de más de $70 \mathrm{~N}$.

- Protector plástico de la malla durante la inserción.
- Sistema de inserción metálico para la colocación desde vagina hacia abdomen. Existe también un dispositivo para la colocación de la malla por vía transobturatriz "in-out" (TVT-O) o por vía retropúbica desde abdomen hacia vagina.

El sistema TVT se utiliza desde al año 1996, año en el que fue descrito por Ulmsten ${ }^{30}$ existiendo una dilatada experiencia en todo el mundo. Desde esa fecha se han implantado más de 500.000 dispositivos. En trabajos recientes se ha publicado la experiencia y resultados con TVT tras 7 años desde la cirugía. Existen también infinidad de estudios publicados por grupos europeos, americanos, etc. recopilando sus resultados en la corrección de la IUE con este tipo de banda suburetral. Esta circunstancia resulta de una enorme importancia y uno de los valores más importantes a tener en 


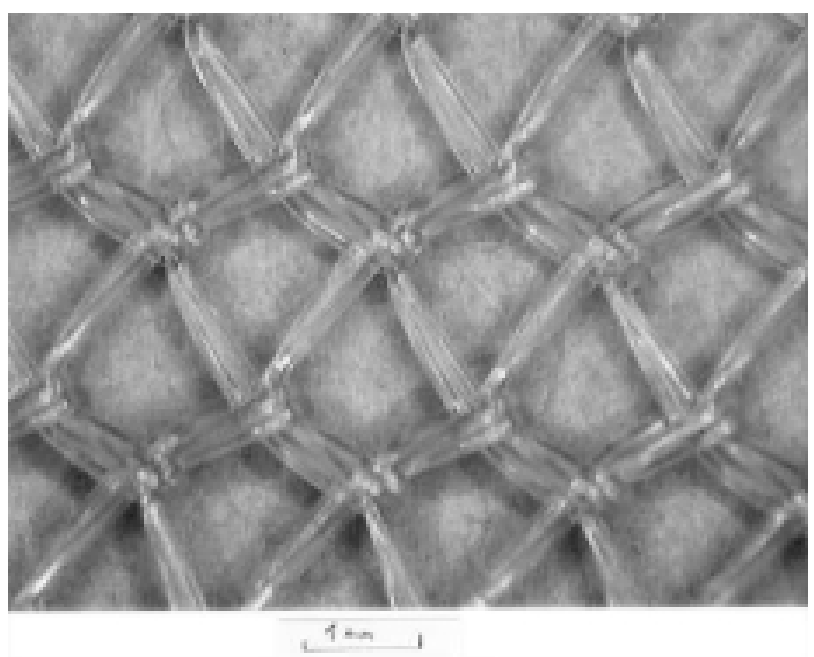

FIGURA 4. Malla de TVT.

cuenta a la hora de seleccionar el dispositivo a utilizar. La experiencia con cualquiera de las demás mallas es infinitamente menor y no se dispone de series publicadas con un número de pacientes y con un tiempo de seguimiento suficiente para poder comparar los resultados con TVT $^{31,32}$. No creemos que se puedan extrapolar los resultados conseguidos con TVT a cualquier otro sistema libre de tensión. Como se ha podido comprobar, las diferencias en las características de la malla o en el sistema de implantación hacen que dicha extrapolación carezca de sentido. Los resultados deberán ser analizados para cada sistema en particular sin presuponer que la eficacia alcanzada con uno de ellos va a ser conseguida con cualquier otro.

\subsection{SPARC / MONARC (AMS) (Fig. 5)}

La malla que utiliza el sistema SPARC / MONARC es la más parecida a la del TVT. Cómo ésta, también está fabricada con polipropileno monofilamento, tricotada, de baja densidad (46\%), protegida con una cubierta plástica para facilitar su colocación. La elasticidad de la malla es buena aunque no resulta tan favorable como la del TVT, pudiendo comprobarse en la Figura 3 que para conseguir una elongación de $40 \mathrm{~mm}$ es necesario aplicar una fuerza de al menos $20 \mathrm{~N}$ (el doble de la necesaria con TVT). Una de las mejoras que aporta este dispositivo es que dispone de un hilo de material absorbible (PGA) que permite traccionar del implante en caso de haberlo colocado con excesiva tensión.

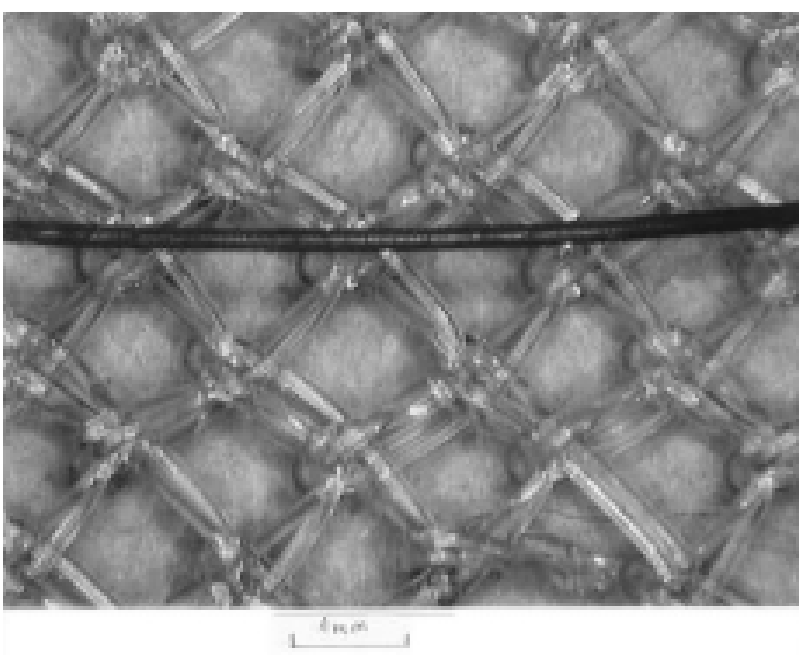

FIGURA 5. Malla del sistema Sparc.

Las principales diferencias con respecto a la malla de Gynecare se encuentran en el sistema de implantación. En este caso la malla se coloca mediante unos insertores que se pasan desde el abdomen hacia la vagina (SPARC) o por vía transobturatriz "out-in" (MONARC). El sistema de inserción tiene el inconveniente de que no es posible liberar la malla de su anclaje a la aguja, de forma que en caso de necesidad (perforación inadvertida de la vejiga, etc.) se deberá utilizar un nuevo dispositivo.

\subsection{IVS (Tyco) (Fig. 6)}

Se trata de una malla de polipropileno multifilamento (Tipo III de Amid), tricotada, de alta densidad de material (10\%). El "efecto velcro" es prácticamente nulo y por ello resulta innecesaria la cubierta plástica protectora, de la que carece. La malla se implanta mediante un abordaje retropúbico.

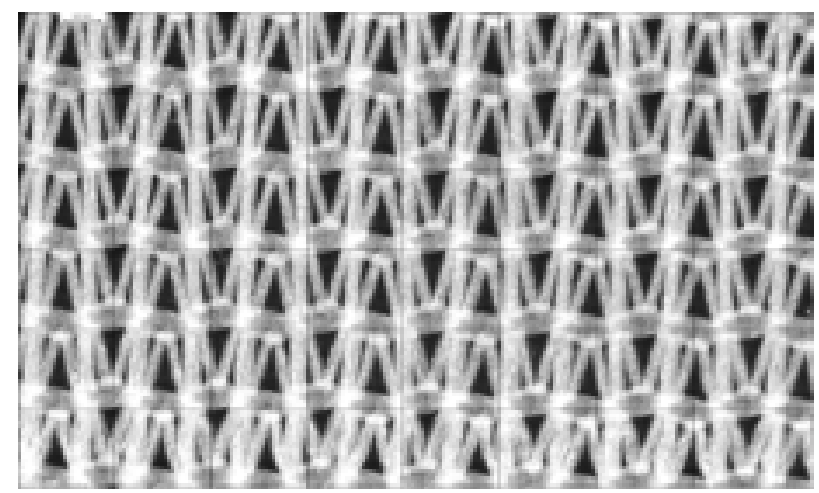

FIGURA 6. Malla IVS. 


\subsection{URATAPE / OBTAPE (Porges) (Fig. 7)}

La malla de Porges se fabricó inicialmente con dos componentes: los extremos eran de polipropileno monofilamento termocoagulado y la parte central, sobre la que se apoya la uretra, de silicona. Este elemento central se caracterizaba por su elevada densidad y carácter microporoso habiendo sido eliminado recientemente. El sistema uratape se coloca por vía retropúbica y el obtape por vía transobturadora.

\subsection{URETEX (Bard) (Fig. 8)}

Se trata de una malla de polipropileno monofilamento, tricotada, de baja densidad (64\%) para colocación por vía retropúbica.

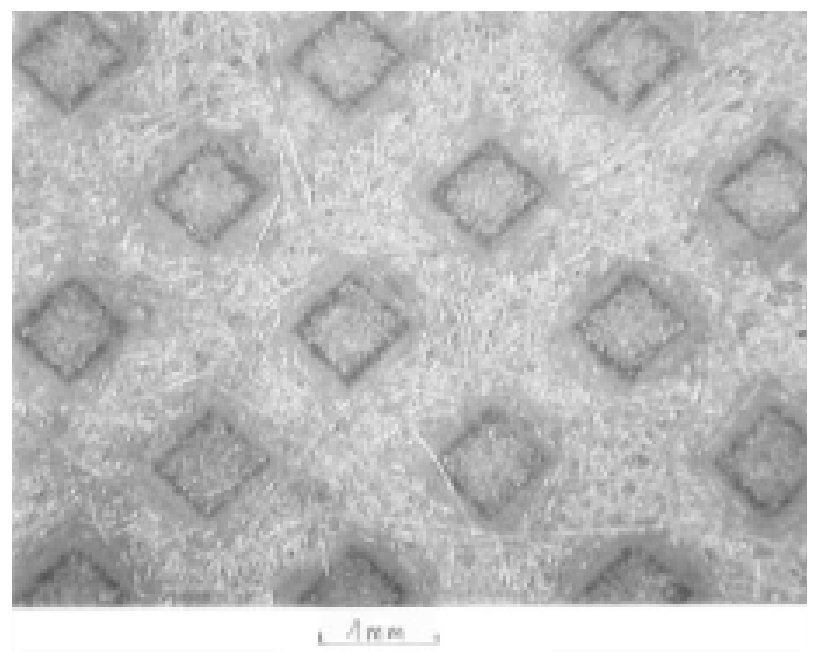

FIGURA 7. Malla de Uratape/Obtape.

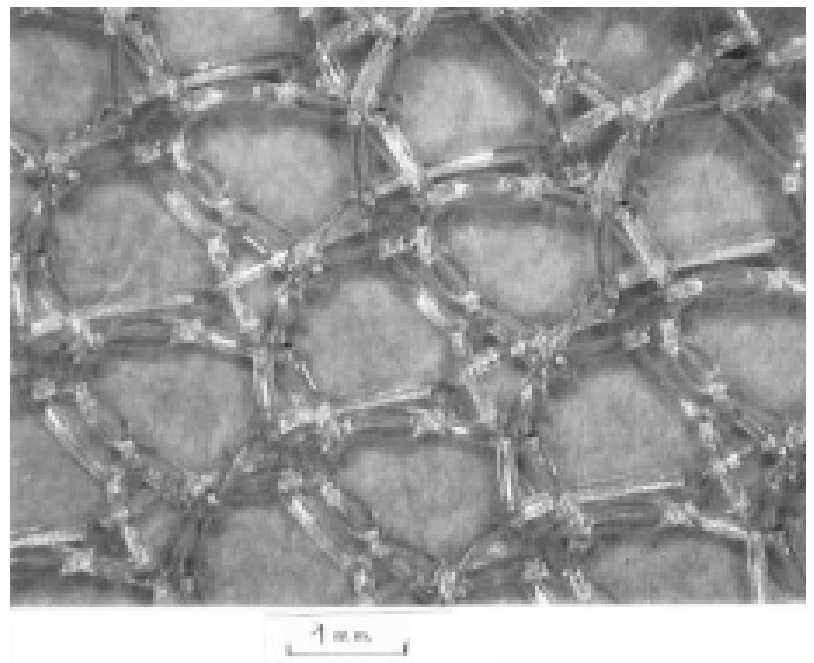

FIGURA 8. Malla Uretex.

\section{REFERENCIAS}

1. BIRCH C, FYNES MM.: The role of synthetic and biological prostheses in reconstructive pelvic floor surgery. Curr Opin Obstet Gynecol 2002a oct; 14 (5): 527-535.

2. AMID PK, SHULMAN AG, LICHTENSTEIN IL, HAKAKHA M.: Biomaterials for abdominal wall hernia surgery and principles of their applications. Langenbecks Arch Chir 1994; 379 (3): 168-171.

3. SANDERS JE, STILES CE, HAYES CL.: Tissue response to single-polymer fibers of varying diameters: evaluation of fibrous encapsulation and macrophage density. J Biomed Mater Res 2000 oct; 52 (1): 231-237.

4. WELTY G, KLINGE U, KLOSTERHALFEN B, KASPERK R, SCHUMPELICK V.: Functional impairment and complaints following incisional hernia repair with different polypropylene meshes. Hernia 2001 sep; 5 (3): 142-147.

5. BELLÓN JM, CONTRERAS LA, BUJÁN J, CARRERA-SAN MARTÍN A.: The use of biomaterials in the repair of abdominal wall defects: a comparative study between polypropylene meshes (Marlex) and a new polytetrafluoroethylene prosthesis (Dual Mesh). J Biomater Appl 1997 oct; 12 (2): 121-135.

6. COSSON M, BOUKERROU M, LOBRY P, CREPIN G, EGO A.: Mechanical properties of biological or synthetic implants used to treat genital prolapse and stress incontinence in women: what is the ideal material?. J Gynecol Obstet Biol Reprod (Paris) 2003 jun; 32 (4): 321-328.

7. GRECA FH, DE PAULA JB, BIONDO-SIMOES ML, DA COSTA FD, DA SILVA AP, TIME S, MANSUR A.: The influence of differing pore sizes on the biocompatibility of two polypropylene meshes in the repair of abdominal defects. Experimental study in dogs. Hernia 2001 jun; 5 (2): 59-64.

8. JUNGE K, KLINGE U, PRESCHER A, GIBONI P, NIEWIERA M, SCHUMPELICK V.: Elasticity of the anterior abdominal wall and impact for reparation of incisional hernias using mesh implants. Hernia 2001 sep; 5 (3): 113-118.

9. KLINGE U, KLOSTERHALFEN B, CONZE J, LIMBERG W, OBOLENSKI B, OTTINGER AP, SCHUMPELICK V.: Modified mesh for hernia repair that is adapted to the physiology of the abdominal wall. Eur J Surg 1998 dec; 164 (12): 951-960.

10. DIETZ HP, VANCAILLIE P, SVEHLA M, WALSH W, STEENSMA AB, VANCAILLIE TG.: Mechanical properties of urogynecologic implant materials. Int Urogynecol J Pelvic Floor Dysfunct 2003 oct; 14 (4): 239-243; discussion 243.

11. DEBODINANCE P, DELPORTE P, ENGRAND JB, BOULOGNE M.: Development of better tolerated prosthetic materials: applications in gynecological surgery. J Gynecol Obstet Biol Reprod (Paris). 2002a oct; 31 (6): 527-540.

12. CERVIGNI M, NATALE F.: The use of synthetics in the treatment of pelvic organ prolapse. Curr Opin Urol 2001 jul; 11 (4): 429-435.

13. KLINGE U, JUNGE K, SPELLERBERG B, PIROTH C, KLOSTERHALFEN B, SCHUMPELICK V.: Do multifilament alloplastic meshes increase the infection rate? Analysis of the polymeric surface, the 
bacteria adherence, and the in vivo consequences in a rat model. J Biomed Mater Res 2002; 63 (6): 765-771.

14. FOURNIER N, DOILLON CJ.: Biological moleculeimpregnated polyester: an in vivo angiogenesis study. Biomaterials 1996 sep; 17 (17): 1659-1665.

15. FALCONER C, SODERBERG M, BLOMGREN B, ULMSTEN U.: Influence of different sling materials on connective tissue metabolism in stress urinary incontinent women. Int Urogynecol $J$ Pelvic Floor Dysfunct 2001; 12 Suppl 2: S19-S23.

16. JUNGE K, KLINGE U, ROSCH R, KLOSTERHALFEN B, SCHUMPELICK V.: Functional and morphologic properties of a modified mesh for inguinal hernia repair. World J Surg 2002 dec; 26 (12): 1472-1480.

17. ABBAS SHOBEIRI S, GARELY AD, CHESSON RR, NOLAN TE.: Recognition of occult bladder injury during the tension-free vaginal tape procedure. Obstet Gynecol 2002 jun; 99 (6): 1067-1072.

18. KOBASHI KC, GOVIER FE.: Perioperative complications: the first 140 polypropylene pubovaginal slings. J Urol 2003 nov; 170 (5): 1918-1921.

19. VIERHOUT ME.: Severe hemorrhage complicating tension-free vaginal tape (TVT): a case report. Int Urogynecol J Pelvic Floor Dysfunct 2001; 12 (2): 139-140.

20. WALTERS MD, TULIKANGAS PK, LASALA C, MUIR TW.: Vascular injury during tension-free vaginal tape procedure for stress urinary incontinence. Obstet Gynecol 2001 nov; 98 (5 Pt 2): 957-959.

21. ZILBERT AW, FARRELL SA.: External iliac artery laceration during tension-free vaginal tape procedure. Int Urogynecol J Pelvic Floor Dysfunct 2001; 12 (2): 141-143.

22. FOURIE T, COHEN PL.: Delayed bowel erosion by tension-free vaginal tape (TVT). Int Urogynecol $J$ Pelvic Floor Dysfunct 2003 nov; 14 (5): 362-364.

23. LEBOEUF L, TELLEZ CA, EAD D, GOUSSE AE.: Complication of bowel perforation during insertion of tension-free vaginal tape. J Urol 2003 oct; 170 (4 Pt 1): 1310; discussion 1310-1311.
24. DAHER N, BOULANGER JC, ULMSTEN U.: Prepubic TVT: an alternative to classic TVT in selected patients with urinary stress incontinence. Eur $J$ Obstet Gynecol Reprod Biol 2003 apr 25; 107 (2): 205-207.

25. DEVAL B, LEVARDON M, SAMAIN E, RAFII A, CORTESSE A, AMARENCO G, CIOFU C, HAAB F.: A French multicenter clinical trial of SPARC for stress urinary incontinence. Eur Urol 2003 aug; 44 (2): 254-258; discussion 258-259.

26. DELORME E.: Transobturator urethral suspension: mini-invasive procedure in the treatment of stress urinary incontinence in women. Prog Urol 2001 dec; 11 (6): 1306-1313.

27. DE LEVAL J.: Novel surgical technique for the treatment of female stress urinary incontinence: transobturator vaginal tape inside-out. Eur Urol 2003 dec; 44 (6): 724-730.

28. HERMIEU JF, MESSAS A, DELMAS V, RAVERY V, DUMONCEAU O, BOCCON-GIBOD L.: Bladder injury after TVT transobturator. Prog Urol 2003 feb; 13 (1): 115-117.

29. DELORME E, DROUPY S, DE TAYRAC R, DELMAS V.: In Process Citation. Prog Urol 2003 sep; 13 (4): 656-659.

30. ULMSTEN U, HENRIKSSON L, JOHNSON P, VARHOS G.: An ambulatory surgical procedure under local anesthesia for treatment of female urinary incontinence. Int Urogynecol $J$ Pelvic Floor Dysfunct 1996; 7 (2): 81-85; discussion 85-86.

31. HERMIEU JF, MILCENT S.: In Process Citation. Prog Urol 2003 sep; 13 (4): 636-647.

32. BEZERRA CA, BRUSCHINI H.: Suburethral sling operations for urinary incontinence in women. Cochrane Database Syst Rev 2001; (3): CD001754.

Dr. I. Galmés Belmonte

Po de la Castellana, $129-7^{\circ} \mathrm{B}$

28046 Madrid

(Trabajo recibido el 5 abril de 2004) 